\title{
Study on Two-phase Flow Mechanisms in Nanopore Considering Microcosmic Deformation and Dynamic Capillary Force
}

\author{
Xiangji Dou (D, Cong Lin, Sujin Hong, Pengfei Zhu, An Wang, and Guodong Qi \\ School of Petroleum Engineering, Changzhou University, Changzhou 213164, China \\ Correspondence should be addressed to Guodong Qi; qiguodong77@163.com
}

Received 10 December 2021; Revised 4 January 2022; Accepted 10 January 2022; Published 8 February 2022

Academic Editor: Zhiming Chen

Copyright (C) 2022 Xiangji Dou et al. This is an open access article distributed under the Creative Commons Attribution License, which permits unrestricted use, distribution, and reproduction in any medium, provided the original work is properly cited.

Coring experiments show that nanopores are extensively distributed in shale oil reservoirs and tend to be deformed when a significant pressure variation exists, and thus the dynamic capillary force phenomenon and flow mechanisms in nanopores can be significantly changed. To characterize the two-phase flow mechanisms in nanopores influenced by the synergistic effect of microcosmic pore deformation and dynamic capillary force, models based on Gassmann's theory are established to describe the variations of pore radius and roughness in a dynamic pressure field. And then, innovative methods to quantify the dynamic capillary force phenomenon under comprehensive influence of pore size, roughness, and pressure are developed. Meanwhile, mathematical models, considering the effect of the pore deformation and dynamic capillary force, are furtherly derived to characterize the water-oil two-phase flow behavior for relatively large nanopores in shale oil reservoir, which can be used to investigate the influence of the vital parameters. The results indicate that the dynamic capillary force phenomenon turns out to be more significant when variations of pore structure and pressure are considered simultaneously. Moreover, the pore deformation and dynamic capillary force caused by pressure change can exert remarkable synergistic influence on the transport capacity for typical flow modes. Bulk modulus is one of the key factors to determine the degree of influence. An optimal pressure can be obtained to coordinate the competitive effect of seepage channel and capillary force for water-drive-oil mode with limited driving force. Based on that, emphasis should be placed on pressure control during the shale oil development process. This work theoretically underpins the quantitative characterization and the analysis of two-phase flow in shale reservoirs at the nanopore scale.

\section{Introduction}

Over the past few years, shale oil has evolved as a vital part of the world's energy system and is expected to be a remarkable growth point for future crude oil production. During the development of shale oil, considerable fracturing fluid should be injected into the shale oil reservoir to enhance the production of shale oil. Due to the fracturing fluid and the shale oil in the reservoir, two-phase flow (oil-water) phenomenon is widely found in the shale oil reservoir. Moreover, with the continuous extraction of the shale oil and the fracturing fluid, the stress conditions of the rocks and fluids in the reservoir will generally vary, which can lead to the deformation of pore microstructure as well as the change of interphase forces. As a result, the two-phase flow in the nanopore of shale oil reservoir can be relatively complex, which is a critical theoretical issue in the shale oil development.

The previous study on flow mechanisms of the shale oil reservoir primarily highlights single-phase flow. By applying the molecular dynamics method, Wang et al. [1] reported that the quantitative description of bulk phase transport could be achieved based on the optimized Navier-Stokes equation for nanopores with small pore sizes. Cui et al. [2] and Su et al. [3] furtherly proposed mathematical models for bulk-phase transport. It is worth noting that there are significant differences between the flow in the small nanopore and the large nanopore. For small nanopore (i.e., pore radius less than $10 \mathrm{~nm}$ ), the Knudsen number is large and the velocity slip cannot be ignored. However, for larger nanopores, the effect of the velocity slip is limited and the fluid flow is more consistent with the Navier-Stokes equation. 
Although relevant studies [4-6] are critical to shale oil microscopic exploration, two-phase transports have not been be taken into consideration.

Most studies on two-phase (oil-water) transport modes in shale reservoirs mainly focus on the macroscopic scale. Das et al. [7] and Gruener and Huber [8] analyzed the transport characteristics under capillary forces and explored the replacement effect of water on crude oil. Their researches show that interfacial tension can significantly affect the transport characteristics. Zhou et al. [9] investigated the twophase (oil-water) macroscopic transport characteristics in shale oil reservoirs and analyzed the main influencing factors. However, all the mentioned studies assumed that the pore size is assumed to be constant and the dynamic capillary force phenomenon caused by the pore deformation is ignored, which results in a less accurate portrayal of the real oil-water two-phase transport mode mechanisms in the nanopores scale.

To clarify the two-phase transport mechanisms under the synergistic effect of microscopic pore deformation and dynamic capillary force, the pore deformation characteristics should be investigated. Common methods on this research currently include laboratory experiment, numerical simulation, and micromechanical analytical equation. To be specific, the laboratory experimental method employed by Wijaya and Sheng [10], Crawford et al. [6], and Kim et al. [11] aims to build the empirical model of the variation of porosity with pore pressure macroscopically by fitting the experimental data, whereas such a type of model still covers certain empirical parameters and exhibits relatively poor applicability to the microscopic scale. The numerical simulation method adopted by Stoxreiter et al. [12, 13] largely complies with the use of finite element techniques to analyze the pore deformation characteristics during triaxial compression. However, the conventional numerical simulation methods applied in the above methods still have some limitations in the application scale and the pore variation is obtained based on the statistical results, which is difficult to accurately describe the deformation of an individual nanopore. Representative analytical equation studies are presented by Lei et al. [14] and Xia et al. [15]. The former mainly adopted the Herz contact deformation theory to determine the deformation volume and tried to establish a quantitative correlation between pore structure and pore pressure. However, results presented by Lei may be more applicable in sandstone instead of shale. The latter derived the relationship between the volume strain of shale nanopore and pore pressure, which is highly conducive to the calculations of shale nanopore deformation.

Under dynamic capillary force, the research complies with the analysis of wettability and fluid interfacial tension. Bennion and Bachu [16] suggested that the variation of interfacial tension between fluids with stress conditions is a vital factor of the two-phase seepage mechanism under dynamic stress fields and thus its effect should be analyzed and verified more specifically. Marghmaleki et al. [17-21] examined the correlation among rock wettability, fluid interfacial tension, capillary force, pressure, and pore throat structure. However, the correlation was not extended to examine the effect of the above interphase interaction variations on the two-phase seepage mechanism.

Although numerous researches have been done on the pore structure, interphase interaction, and flow mechanisms in macroscale [22-26], mathematical models capable of accurately characterizing the pore deformation, dynamic capillary force phenomenon, and oil-water two-phase transport at the nanopore scale for shale oil are still missing [27-31]. To solve this problem, Gassmann's method is modified to describe the change of pore radius and roughness in the dynamic pressure field for elastic deformation. And then, the wettability and interfacial tension variation characteristics are furtherly analyzed under the synergistic effect of pore size, roughness, and pressure, which reveals the effect of the dynamic capillary force. Subsequently, the mathematical models of two-phase flow (oil-water) in relatively large nanopores under two types of transport modes, i.e., oil-in-water and water-drive-oil, are built, respectively. Based on the abovementioned research, an investigation is conducted on the effect of pore deformation, dynamic capillary force, pressure, bulk modulus, flow mode, and driving force on the transport mechanisms in nanopores, thereby underpinning the characterization of two-phase migration in shale reservoirs at the microscopic scale.

\section{Microscopic Deformation of Nanopore}

During the development of unconventional reservoirs (e.g., shale oil), the pore pressure of the reservoir will vary as impacted by the flow and output of fluids, thereby leading to the variation of the effective stress, and then causing the microscopic deformation of the pore, which would significantly influence the two-phase transport mechanisms.

For the reservoir under original formation pressure, a stress balance could be found. However, with the withdrawal of fluids, the formation pressure would decrease, and the balance may be broken, and then pores would be deformed. If we assume that the deformation is elastic, the change in the pore volume can be expressed in the form of equation (1).

$$
\Delta V_{\phi}=V_{\phi-0} \varepsilon_{\phi},
$$

where $\Delta V_{\phi}$ is the variation of pore volume, $\mathrm{nm}^{3} ; V_{\phi-0}$ represents the pore volume under the surface pressure, $\mathrm{nm}^{3}$; $\varepsilon_{\phi}$ denotes pore volume deformation ratio, dimensionless.

According to the Gassmann equation [15], the relationship between porous media volume deformation ratio, solid skeleton deformation ratio, and pore volume deformation ratio could be described as:

$$
\varepsilon_{t}=\phi \varepsilon_{\phi}+(1-\phi) \varepsilon_{s},
$$

where $\phi$ expresses porosity, dimensionless; $\varepsilon_{t}$ is the porous media volume deformation ratio, dimensionless; $\varepsilon_{\phi}$ denotes pore volume deformation ratio, dimensionless; $\varepsilon_{s}$ represents solid skeleton deformation ratio, dimensionless.

Therefore, the pore volume deformation ratio could be rewritten as: 


$$
\varepsilon_{\phi}=\frac{\left[\varepsilon_{t}-(1-\phi) \varepsilon_{s}\right]}{\phi} .
$$

Moreover, the average normal stress acting on the porebearing rock can be defined as [32],

$$
\Delta \bar{\sigma}=(1-\phi) K_{s} \varepsilon_{s}-\phi \Delta p_{f},
$$

where $\Delta \bar{\sigma}$ denotes average normal stress acting on the pore-bearing rock, $\mathrm{MPa} ; K_{s}$ is bulk modulus of solid skeleton, $\mathrm{MPa} ; \varepsilon_{s}$ represents solid skeleton deformation ratio, dimensionless; $\Delta p_{f}$ expresses change of fluid pressure, MPa.

The bulk deformation modulus of solid skeleton can be expressed as:

$$
K_{s}=\frac{K}{1-\alpha}
$$

where $\alpha$ is the Bolt Coefficient, dimensionless.

Thus, the solid skeleton deformation ratio could be obtained by:

$$
\varepsilon_{s}=\frac{\left(\Delta \bar{\sigma}+\phi \Delta p_{f}\right) \cdot(1-\alpha)}{[(1-\phi) K]} .
$$

The average normal stress acting on the pore-bearing rock can also be described as:

$$
\Delta \bar{\sigma}=K \varepsilon_{t}-\alpha \Delta p_{f} .
$$

Therefore, the porous media volume deformation ratio could be deduced as:

$$
\varepsilon_{t}=\frac{\left(\Delta \bar{\sigma}+\alpha \Delta p_{f}\right)}{K} .
$$

Then, the pore volume deformation ratio could be obtained by substituting equations (6) and (8) into equation (2).

$$
\varepsilon_{\phi}=\frac{\left(\alpha \Delta p_{f}-\phi \Delta p_{f}+\alpha \Delta \bar{\sigma}+\alpha \phi \Delta p_{f}\right)}{(\phi \cdot K)} .
$$

Based on equations (1) and (9), the variation of pore volume can be obtained:

$$
\Delta V_{\phi}=\frac{V_{\phi-0}\left(\alpha \Delta p_{f}-\phi \Delta p_{f}+\alpha \Delta \bar{\sigma}+\alpha \phi \Delta p_{f}\right)}{(\phi \cdot K)} .
$$

And then, the pore volume after deformation can be obtained:

$$
\begin{aligned}
V_{\phi-r} & =V_{\phi-0}-\Delta V_{\phi} \\
& =V_{\phi-0}\left[1-\frac{\left(\alpha \Delta p_{f}-\phi \Delta p_{f}+\alpha \Delta \bar{\sigma}+\alpha \phi \Delta p_{f}\right)}{(\phi \cdot K)}\right]
\end{aligned}
$$

where $V_{\phi-r}$ is the pore volume for a certain pressure, $\mathrm{nm}^{3}$.

Moreover, the pore radius under the initial formation pressure can be expressed as:

$$
r_{i}=\sqrt{\frac{V_{\phi-i}}{\pi L}}=r_{0} \sqrt{1-\frac{\alpha \Delta \bar{\sigma}}{(\phi K)}}
$$

where $r_{i}$ denotes the pore radius under the initial formation pressure, $\mathrm{nm} ; r_{i}$ is the pore radius under the surface pressure, $\mathrm{nm}$; and $L$ represents the pore length, $\mathrm{nm}$.

With the variation of formation pressure, the pore radius under a certain pressure is presented as follows:

$$
\begin{aligned}
r & =\sqrt{\frac{V_{\phi-r}}{\pi L}} \\
& =r_{0} \sqrt{1-\frac{\left[\alpha\left(p_{i}-p\right)-\phi\left(p_{i}-p\right)+\alpha \Delta \bar{\sigma}+\alpha \phi\left(p_{i}-p\right)\right]}{(\phi \cdot K)} .} .
\end{aligned}
$$

Accordingly, during the pore deformation, the correlation between the pore diameter and the initial pore diameter is written as:

$$
r=r_{i} \frac{\sqrt{K \phi-\left[\alpha\left(p_{i}-p\right)-\phi\left(p_{i}-p\right)+\alpha \Delta \bar{\sigma}+\alpha \phi\left(p_{i}-p\right)\right]}}{\sqrt{K \phi-\alpha \Delta \bar{\sigma}}} .
$$

With the above equations, the pore radius under different pressures can be calculated based on the known pore pressure and other critical parameters.

It is worth noting that with the variation of formation pressure, the inner surface roughness of the pore is not a constant. In accordance with the compression deformation theory, the height deformation (which is negative) at the protrusion of the inner surface is written as:

$$
\Delta a=\frac{\Delta p}{E_{s}}=\frac{p-p_{i}}{E_{s}},
$$

where $\Delta a$ is the variation of rough protrusion height, $\mathrm{nm} ; E_{s}$ is the compression modulus, MPa.

The correlation between the compression modulus and the elastic modulus is written as:

$$
E_{s}=E \cdot \frac{1-v}{(1+v) \cdot(1-2 v)}
$$

w:ere $E$ is the compression modulus, $\mathrm{MPa} ; v$ is the Poisson's ratio, dimensionless.

The correlation between the elastic modulus and the bulk modulus is written as:

$$
E=3(1-2 v) K_{s}=\frac{3(1-2 v) K}{1-\alpha} .
$$

And thus, the compression modulus can be furtherly expressed as follows:

$$
E_{s}=\frac{3 K(1-v)}{(1-\alpha)(1+v)} .
$$

The height of the protrusion after deformation can be written in the form of equation (19). 


$$
a=a_{i}+\Delta a=a_{i}+\frac{\left(p-p_{i}\right)(1-\alpha)(1+v)}{3 K(1-v)} .
$$

\section{Dynamic Capillary Force Effect}

For the shale oil reservoir, capillary force is vital due to the extensive distribution of nanopores. However, the capillary force could not be regarded as a constant due to the synthetical impact of pore deformation and pressure variation. Moreover, the change of capillary force appears commonly in field production as well as in the experimental testing, which could be defined as the effect of the dynamic capillary force.

The pore deformation can lead to the variation of molecular spacing between fluids and solids, resulting in the variation of interphase interaction parameters, such as microscopic wettability and interfacial tension at the nanopore scale. Wang et al. [1] calculated the wetting angle under different pore diameter conditions by the molecular dynamic method. The results show that for the nanopore, the wetting angle shows dynamic change with pore radius. According to the research [1], a quantitative relationship between the wetting angle and the pore radius was regressed and constructed as follows:

$$
\theta=\theta_{i}+13.115 \ln \left(\frac{r}{r_{i}}\right)
$$

where $\theta$ is the contact angle of a certain pore radius; $\theta_{i}$ is the contact angle for the initial condition.
With the variation of the pore diameter, the oil-water interfacial tension also varies dynamically. Assuming that the change ratio of water droplet diameter and pore diameter within the pore diameter are equal, the variation of interfacial tension with pore diameter can be calculated by using the Lu-Jiang model [33], which is shown in equation (21).

$$
\gamma(r)=\gamma_{\infty}\left(1-\frac{1}{4 r / h-1}\right) \exp \left(-\frac{2 S_{b}}{3 R} \frac{1}{4 r / h-1}\right),
$$

where $\gamma$ is the interfacial tension, $\mathrm{N} / \mathrm{m} ; \gamma_{\infty}$ is the interfacial tension for the pore with the radius tending to infinite, $\mathrm{N} / \mathrm{m}$; $R$ is the ideal gas constant, dimensionless; $E_{0}$ is the enthalpy of vaporization, $\mathrm{kJ} / \mathrm{mol}$; $T_{b}$ is the boiling point, $K$.

In accordance with the above equations, a quantitative relationship between the interfacial tension and the initial interfacial tension considering the pore deformation can be furtherly established:

$$
\begin{aligned}
\gamma(r)= & \gamma\left(r_{i}\right)\left[\frac{(4 r-2 h)\left(4 r_{i}-h\right)}{(4 r-h)\left(4 r_{i}-2 h\right)}\right] \\
& \cdot \exp \left[\frac{2 S_{b}}{3 R}\left(\frac{1}{4 r_{i} / h-1}-\frac{1}{4 r / h-1}\right)\right] .
\end{aligned}
$$

And then, the capillary force can be expressed as follows:

$$
p_{c}=-\frac{2 \gamma(r) \cos \theta(r)}{r} \text {. }
$$

Substituting equations (21) and (22) into equation (23), the capillary force considering the single effect of the deformation can be obtained:

$$
\begin{aligned}
p_{c}= & -\left\{\frac{2 \gamma\left(r_{i}\right)\left[(4 r-2 h)\left(4 r_{i}-h\right) /(4 r-h)\left(4 r_{i}-2 h\right)\right] \cdot \exp \left[\left(2 S_{b} / 3 R\right)\left(\left(1 /\left(4 r_{i} / h-1\right)\right)-t(1 /(4 r / h-1))\right)\right]}{r}\right\} \\
& \cdot \cos \left[\theta_{i}+13.115 \ln \left(\frac{r}{r_{i}}\right)\right] .
\end{aligned}
$$

Considerable experimental studies have been conducted to investigate the effect of pressure on the wetting angle and surface tension. Moreover, by analyzing the relevant experimental results, the following correlation between the wetting angle for the certain pressure and the initial wetting angle (the contact angle corresponding to the original formation pressures) can be yielded,

$$
\theta=\theta_{i} \cdot\left(\frac{p}{p_{i}}\right)^{\tau}
$$

where $\tau$ denotes the wettability modulus, dimensionless, which generally changes from 0.2 to 0.3 .

Besides, according to the results presented by Sarmadivaleh, the interfacial tension at a specific pressure can be expressed by exploiting the interfacial tension corresponding to the initial pressure.

$$
\gamma=\gamma_{i} \cdot\left(\frac{p}{p_{i}}\right)^{\xi}
$$

where $\xi$ denotes the interfacial modulus, dimensionless.

It is worth noting that wettability is impacted by pore diameter and pressure, and the contact angle changes dynamically with surface roughness. According to the model of Wenzel and equation (19), the contact angle influenced by roughness can be expressed as:

$$
\cos \theta=\left[1+\frac{2\left(p-p_{i}\right)(1-\alpha)(1+v)}{3 K(1-v) \lambda}\right] \cos \theta_{e},
$$

where $\theta_{e}$ denotes the intrinsic contact angle of the solid surface; $\lambda$ represents the period distance of protrusion, $\mathrm{nm}$.

By complying with equation (27), the contact angle under certain pressure and the initial pressure can be expressed as follows: 
$\theta=\arccos \left\{\left\{\left[a_{i}+\frac{\left(p-p_{i}\right)(1-\alpha)(1+v)}{3 K(1-v)}\right]+\lambda\right\} \cdot \frac{\cos \theta_{i}}{\left(a_{i}+\lambda\right)}\right\}$.

As mentioned above, the wetting angle and interfacial tension are affected by the pore diameter and the pore pressure while the variation of pore diameter is the result of the variation of the pore pressure. Then, the variation of interphase interaction in nanopores is usually complex and the effect of the pore deformation variation and pressure variation should be considered to construct a mathematical model of interphase interaction in the dynamic pressure field.

The mathematical model of the contact angle variation under the synergistic effect of stress-induced and pore pressure-induced can be expressed as follows:

$$
\theta=\left\{\theta_{i}+13.115 \ln \left(1-\frac{[(1+\phi)+(1-\phi) v] \cdot p_{\mathrm{eff}}}{E(1-\phi)}\right)\right\}\left(\frac{p}{p_{i}}\right)^{\tau} .
$$

When the surface roughness is furtherly considered, equation (29) can be rewritten as follows:

$\theta=\arccos \left\{\left\{2\left[a_{i}+\frac{\left(p-p_{i}\right)(1-\alpha)(1+v)}{3 K(1-v)}\right]+\lambda\right\} \cdot \frac{\cos \theta_{s}}{\left(a_{i}+\lambda\right)}\right\}$,

where $\theta_{s}$ could be described as

$$
\theta_{s}=\left\{\theta_{i}+13.115 \ln \left\{1-\frac{[(1+\phi)+(1-\phi) v] \cdot p_{\mathrm{eff}}}{E(1-\phi)}\right\}\right\}\left(\frac{p}{p_{i}}\right)^{\tau} .
$$

In terms of the interfacial tension from the existing studies, the mathematical model of the pore deformation induced by interfacial tension change can be expressed as:

$$
\begin{aligned}
\gamma(r)= & \gamma\left(r_{i}\right)\left[\frac{(4 r-2 h)\left(4 r_{i}-h\right)}{(4 r-h)\left(4 r_{i}-2 h\right)}\right] \\
& \cdot \exp \left[\frac{2 S_{b}}{3 R}\left(\frac{1}{4 r_{i} /(h-1)}-\frac{1}{4 r /(h-1)}\right)\right] .
\end{aligned}
$$

The interfacial tension change induced by the pore pressure field can be expressed as

$$
\gamma=\gamma_{i} \cdot\left(\frac{p}{p_{i}}\right)^{\xi} .
$$

Accordingly, the mathematical model of interfacial tension that integrates the synergistic effect of the deformation field and the pore pressure field can be written as

$$
\begin{aligned}
\gamma= & {\left[\gamma_{i} \cdot\left(\frac{p}{p_{i}}\right)^{\xi}\right] \cdot\left\{\left[\frac{\left(4 r_{c}-2 h\right)\left(4 r_{c i}-h\right)}{\left(4 r_{c}-h\right)\left(4 r_{c i}-2 h\right)}\right]\right.} \\
& \left.\cdot \exp \left[\frac{2 S_{b}}{3 R}\left(\frac{1}{4 r_{c i} /(h-1)}-\frac{1}{4 r_{c} /(h-1)}\right)\right]\right\} .
\end{aligned}
$$

And, the capillary force can be expressed as

$$
\begin{aligned}
p_{c}= & \left\{2 \gamma_{i} \cdot\left(\frac{p}{p_{i}}\right)^{\xi} \cdot\left\{\left[\frac{\left(4 r_{c}-2 h\right)\left(4 r_{c i}-h\right)}{\left(4 r_{c}-h\right)\left(4 r_{c i}-2 h\right)}\right] \cdot \exp \left[\frac{2 S_{b}}{3 R}\left(\frac{1}{4 r_{c i} /(h-1)}-\frac{1}{4 r_{c} /(h-1)}\right)\right]\right\} \cos \theta(r)\right\} \\
& \cdot \frac{\left\{\left[\left(2\left[a_{i}+\left(\left(p-p_{i}\right)(1-\alpha)(1+v) / 3 K(1-v)\right)\right]+b /\left(a_{i}+b\right)\right)\right] \cos \left\{\left\{\theta_{i}+13.115 \ln \left\{1-\left([(1+\phi)+(1-\phi) v] \cdot p_{\text {eff }} / E(1-\phi)\right)\right\}\right\} \cdot\left(p / p_{i}\right)^{\tau}\right\}\right\}}{r} .
\end{aligned}
$$

It could be concluded from equation (35) that due to the pore deformation and the pressure variation, the capillary force for the shale oil reservoir is related to the pore pressure and mechanical properties, which are significantly different from the conventional reservoir.

\section{Oil-Water Two-Phase Flow Model}

During the withdrawal of shale oil, the two-phase (oil-water) flow in the water-wet pore may occur in the following types, namely, oil-in-water mode and water-drive-oil mode. For nanopores with relatively large pore diameters, as indicated from previous studies, the Hagen-Poiseuille equation can be approximately applied for characterization. However, for pores with small diameters (e.g., the radius less than $5 \mathrm{~nm}$ ), the slip length should be revised. Then, to ensure the accuracy of this work, we mainly analyze the pore in which the initial pore diameter is larger than $50 \mathrm{~nm}$.

Figure 1 mainly illustrates the oil-in-water mode which includes three regions from outside to inside (i.e., bound water, free water, and crude oil). Thus, according to the Hagen-Poiseuille equation, the oil phase flow in the pore space can be defined as follows by considering the effect of the capillary force.

$$
q_{o}\left(r_{o-\text { eff }}\right)=G \frac{\Delta p_{o}}{L_{t}} \frac{r_{o-\text { eff }}^{4}}{\mu_{o}},
$$

where $q_{o}$ denotes oil flow rate, $\mathrm{m}^{3} / \mathrm{s} ; G$ represents pore shape correction coefficient, dimensionless; $r_{o-\text { eff }}$ expresses effective radius for oil flow, $\mathrm{m} ; \Delta p_{o}$ is the pressure drop of oil, $\mathrm{MPa} ; L$ is pore length, $\mathrm{m} ; \mu_{o}$ denotes the viscosity for oil, $\mathrm{cp}$. 


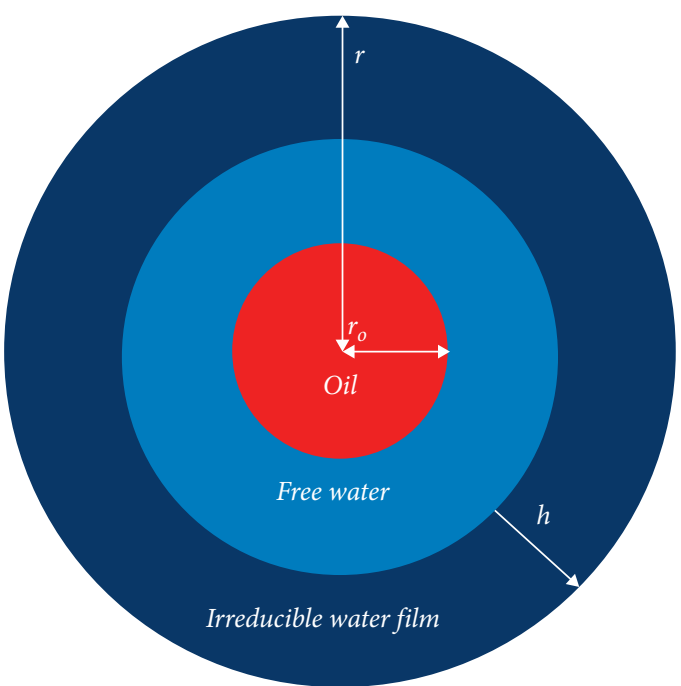

FIgURE 1: Schematic diagram of oil-in-water transport mode.

$$
q_{w}\left(r_{w-\mathrm{eff}}\right)=G \frac{\Delta p_{w}}{L_{t}} \frac{r_{w-\mathrm{eff}}^{4}}{\mu_{w}},
$$

where $q_{w}$ denotes water flow rate, $\mathrm{m}^{3} / \mathrm{s} ; \Delta p_{w}$ represents the pressure drop of water, $\mathrm{MPa} ; r_{w \text {-eff }}$ expresses the effective radius for water flow, $\mathrm{m} ; \mu_{w}$ is the viscosity for water, $\mathrm{cp}$.

Generally, under the oil-in-water transport mode, the capillary force does not directly impact the transport in the flow direction. Moreover, if its indirect effect due to the boundary effect is ignored, the effective transport radius of the oil phase $r_{\text {eff }}$ in two-dimension can be defined as

$$
r_{\mathrm{eff}}=r \sqrt{S_{o}}
$$

where $S_{o}$ denotes oil saturation, dimensionless.

Subsequently, the flow rate of the oil phase in a single pore can be expressed as

Equation (36) can be rewritten as

$$
\begin{aligned}
q_{o}\left(r_{o-\text { eff }}\right) & =G \frac{\Delta p_{m}}{L_{t}} \frac{\left[r \sqrt{\left(1-S_{w}\right)}\right]^{4}}{\mu_{o}} \\
& =G \frac{\Delta p_{m}}{L_{t}} \frac{\left\{r_{i}\left(\sqrt{K \phi-\left[\alpha\left(p_{i}-p\right)-\phi\left(p_{i}-p\right)+\alpha \Delta \bar{\sigma}+\alpha \phi\left(p_{i}-p\right)\right]} / \sqrt{K \phi-\alpha \Delta \bar{\sigma}}\right) \cdot \sqrt{\left(1-S_{w}\right)}\right\}^{4}}{\mu_{o}}
\end{aligned}
$$

where for the flow in a circular tube, $G$ can be written as

$$
G=\frac{\pi}{128}
$$

Based on the Hagen-Poiseuille equation, the water phase flow in the pore can be obtained by considering the effect of capillary forces:

$$
\begin{aligned}
q_{w}\left(r_{w-\mathrm{eff}}\right) & =G \frac{\Delta p_{m}}{L_{t}} \frac{\left[r \sqrt{S_{w}}-h\right]^{4}}{\mu_{w}} \\
& =G \frac{\Delta p_{m}}{L_{t}} \frac{\left\{r_{i}\left(\sqrt{K \phi-\left[\alpha\left(p_{i}-p\right)-\phi\left(p_{i}-p\right)+\alpha \Delta \bar{\sigma}+\alpha \phi\left(p_{i}-p\right)\right]} / \sqrt{K \phi-\alpha \Delta \bar{\sigma}}\right) \cdot \sqrt{S_{w}}-h\right\}^{4}}{\mu_{w}},
\end{aligned}
$$

where $h$ denotes water film thickness, $m$.

Another mode refers to the displacement relationship. During the injection of the fracturing fluid and the well soaking, the water phase (e.g., fracturing fluid) enters into the small pore space by the capillary force and the driving force. And, then the transport phenomenon can be formed and the replacement of crude oil in the small pore space can be obtained, which effectively facilitates the development of shale oil. To be specific, water and oil are the wetting phase and the nonwetting phase, respectively. The transitional zone exists between two phases and the direction of the capillary force ranges from the water phase to the oil phase, as shown in Figure 2. And thus, the pressure difference between the water phase and the oil phase can be formed.

Under water-drive-oil mode, the effective transport radius of the water phase (the flowable free water) and the oil phase are equal, and can be expressed as:

$$
r_{w-\mathrm{eff}}=r_{o-\mathrm{eff}}=r-h .
$$

Subsequently at any time $t$, the flow rate of the water phase and the oil phase can be obtained, respectively: 


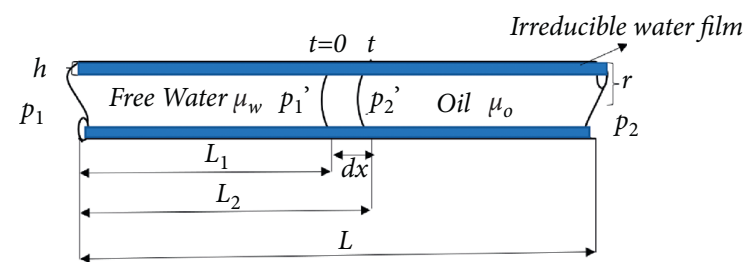

FIGURE 2: Schematic diagram of water-drive-oil transport mode.

$$
\begin{aligned}
& v_{w}=\frac{(r-h)^{2}\left(p_{1}-p_{1}^{\prime}\right)}{8 \mu_{w} L_{2}}, \\
& v_{o}=\frac{(r-h)^{2}\left(p_{2}^{\prime}-p_{2}\right)}{8 \mu_{o}\left(L-L_{2}\right)},
\end{aligned}
$$

where $p_{1}$ denotes starting pressure, $\mathrm{MPa} ; p_{2}$ represents ending pressure, $\mathrm{MPa} ; p_{1}^{\prime}$ is the pressure on the left side of the bend level, MPa; $p_{2}^{\prime}$ expresses pressure on the left side of the bend level; $L_{2}$ is the length of free water zone, $\mathrm{m}$.

The velocity of the water phase is equal to that of the oil phase under the continuous flow, which is equal to that of the two-phase interface movement. And thus, we can obtain

$$
\frac{(r-h)^{2}\left(p_{1}-p_{1}^{\prime}\right)}{8 \mu_{w} L_{2}}=\frac{(r-h)^{2}\left(p_{2}^{\prime}-p_{2}\right)}{8 \mu_{o}\left(L-L_{2}\right)}=\frac{(r-h)^{2}\left(\Delta p+p_{c}\right)}{8\left[\mu_{w} x+\mu_{o}(L-x)\right]}
$$

where $\Delta p=p_{1}-p_{2}$.

Furtherly, the two-phase flow rate under the waterdrive-oil can be yielded:

$$
\begin{aligned}
& v=\frac{(r-h)^{2}\left(\Delta p+p_{c}\right)}{\left.8 \sqrt{\left(\mu_{o} L\right)^{2}-\left(\mu_{o}-\mu_{w}\right)\left[\left((r-h)^{2} t / 4\right)\left(\Delta p+p_{c}\right)+2 \mu_{o} L L_{1}-L_{1}^{2}\left(\mu_{o}-\mu_{w}\right)\right.}\right]} . \\
& \qquad L_{1}=L\left[1-\frac{\left(1-S_{w}\right) r^{2}}{(r-h)^{2}}\right]
\end{aligned}
$$

If we assume that the two-phase fluid transport distance is small in the transient phase, the water saturation at this time can be approximately expressed as

$$
S_{w}=1-\frac{\left(L-L_{1}\right) \cdot(r-h)^{2}}{L \cdot r^{2}}
$$

Substituting equation (47) into equation (45), the instantaneous flow rate of the two-phase fluid can be yielded as

Then, $L_{1}$ can be obtained as follows:

$$
q_{w}=q_{o}=\frac{(r-h)^{4}\left(\Delta p+p_{c}\right)}{\left.8 \sqrt{\left(\mu_{o} L\right)^{2}-\left(\mu_{o}-\mu_{w}\right) \cdot\left\{\left((r-h)^{2} t / 4\right)\left(\Delta p+p_{c}\right)+2 \mu_{o} L\left[1-\left(\left(1-S_{w}\right) r^{2} /(r-h)^{2}\right)\right]-L^{2}\left[1-\left(\left(1-S_{w}\right) r^{2} /(r-h)^{2}\right)\right]^{2}\left(\mu_{o}-\mu_{w}\right)\right.}\right\}} .
$$

The pore diameter, bound water thickness, and capillary force for different pore structures as well as pressure can be obtained by solving equation (48) and then the flow characteristics under the synergistic effect of pore deformation and pressure variation can be furtherly yielded.

\section{Analysis of the Main Control Factors of Two- Phase Transport}

With the mathematical models of two-phase flow (oil-water) in nanopores, the influence of pore deformation, dynamic capillary force, formation pressure, bulk modulus, transport mode, and driving force on the flow mechanisms is analyzed in detail. The basic parameters for analysis are listed in Table 1.

5.1. Effect of Pore Deformation, Dynamic Capillary Force, and Pressure. To investigate the effect of pore deformation, dynamic capillary force, and pressure, four different cases are assumed.
Case 1: the effect of pore deformation and pressure variation is ignored

Case 2: only the direct effect of pore deformation on two-phase flow is considered while the dynamic capillary force phenomenon is ignored

Case 3: the direct effect of pore deformation on twophase flow as well as dynamic capillary force phenomenon caused by pressure variation are considered while the dynamic capillary force phenomenon caused by pore deformation is ignored

Case 4: the synergetic effect of pore deformation and dynamic capillary force phenomenon is considered at the same time

The comparison between assumptions for different cases is shown in Table 2.

By using equation (35), the characteristics of the interfacial force are calculated and compared for different cases. Figures 3-5 present the correlation between the dimensionless wetting angle, interfacial tension, capillary force, and 
TABLE 1: The basic parameters for analysis.

\begin{tabular}{lc}
\hline Parameter & Value \\
\hline Original radius & $50 \mathrm{~nm}$ \\
Roughness initial height & $1 \mathrm{~nm}$ \\
Pore length & $1000 \mathrm{~nm}$ \\
Driving force & $0.5 \mathrm{MPa}$ \\
Water saturation & 0.5 \\
Crude oil viscosity & $3 \mathrm{cp}$ \\
Bound water thickness & $4 \mathrm{~nm}$ \\
Original pressure & $30 \mathrm{MPa}$ \\
\hline
\end{tabular}

TABle 2: Comparison between assumptions for different cases.

\begin{tabular}{cccc}
\hline Case & $\begin{array}{c}\text { Direct effect of } \\
\text { pore deformation } \\
\text { on flow }\end{array}$ & $\begin{array}{c}\text { Dynamic capillary } \\
\text { force caused by } \\
\text { pressure variation }\end{array}$ & $\begin{array}{c}\text { Dynamic } \\
\text { capillary } \\
\text { force caused by } \\
\text { pore deformation }\end{array}$ \\
\hline Case 1 & $\times$ & $\times$ & $\times$ \\
Case 2 & $\sqrt{ }$ & $\times$ & $\times$ \\
Case 3 & $\sqrt{ }$ & $\sqrt{ }$ & $\times$ \\
Case 4 & $\sqrt{ }$ & $\sqrt{ }$ & $\sqrt{ }$ \\
\hline
\end{tabular}

pressure, respectively. It worth noting that both in Case 1 and in Case 2, the capillary force is assumed to be a constant, therefore, only Case 1, Case 3, and Case 4 are compared to make the figures more clear. In Figures 3-5, the dimensionless contact angle is defined as the ratio between the contact angle under certain pressure and the contact angle under the initial pressure. Similarly, the dimensionless interfacial tension is defined as the ratio between the interfacial tension under certain pressure and the interfacial tension under the initial pressure while the dimensionless capillary force is defined as the ratio between the capillary force under certain pressure and the capillary force under the initial pressure.

It could be observed from Figures 3-5 that for Case 1, because the pore deformation and pressure variation are not assumed, the contact angle, interfacial tension, and capillary force remain constant.

However, for Case 3, with the decreasing of the pressure, the dimensionless contact angle gradually decreases (Figure 3) while the dimensionless interfacial tension increases (Figure 4), which significantly leads to the increase of the capillary force (Figure 5) when the decreasing of the dimensionless contact angle and the increasing of the dimensionless interfacial tension are combined with the effect of pore deformation.

Moreover, when the synergistic effect of pore deformation and pressure variation are taken into consideration (Case 4), the decrease of the wetting angle is furtherly observed (Figure 3) while the increase of the interfacial tension can be neglected (Figure 4). The single effect of the pore deformation will lead to the decrease of the interfacial tension, which will aggravate the increase of the capillary force (Figure 5). Therefore, the dynamic capillary force phenomenon is more significant when the synergistic effects of pore deformation and pressure change are considered.
The flow characteristics of different cases are furtherly calculated through equations (39), (41), and (48). Figure 6 presents the correlation between dimensionless flow rate and pore pressure for Case 1 and Case 4 under the oil-in-water transport mode. It should be noted that the driving force, which is the pressure drop from the inlet to the outlet of the nanopore, is assumed to be constant and does not change with the pore pressure during the calculation. The dimensionless flow rate is defined as the ratio between the flow rate under the pressure and the flow rate under the initial pressure.

In Figure 6, we can observe that for the oil-in-water mode, the dimensionless flow rate of oil and water shows a direct proportion to the pore pressure when the pore deformation is considered. For instance, if we assume that the driving force is a fixed value, the oil-phase flow rate remains constant without considering the effect of the pore deformation while the oil-phase flow rate under different pressure varies when the pore deformation is considered. Accordingly, we can conclude that the fluid transport for the oil-inwater mode is significantly influenced by the pore deformation.

Based on equation (48), the dimensionless fluid rates under different pressure for water-drive-oil mode are calculated and shown in Figure 7.

It could be concluded from the comparison between case 1 and case 2 that the single effect of pore deformation can lead to a significant drop in production. Moreover, compared with case 2, the fluid flow rate under the identical pressure goes up with the increase of the capillary force exerted by the reduction of the pressure (case 3), which demonstrates that the transport capacity of fluids is greatly improved. When the variation of capillary force caused by the pore deformation is additionally taken into consideration (case 4), the transport capacity is furtherly enhanced. However, compared with case 1 , the co-effect of pore deformation and the dynamic capillary force is considered (case 4), and the fluid flow rate under the identical pressure is significantly decreased, which is established by using the parameters in Table 1 . In addition, the flow rate variation under the co-effect of the pore deformation and the capillary force may show different trends with the change of the driving force and other parameters, which will be elaborated in the following discussion of this paper.

5.2. Effect of Bulk Modulus. The bulk modulus determines the magnitude of pore deformation under identical pressure and exerts direct or indirect effect on the interphase interaction and transport mode. Based on equation (35), an investigation is furtherly conducted on the comparison between dimensionless contact angle, interfacial tension, pore size capillary force, and bulk modulus when the pore pressure decreases to $5 \mathrm{MPa}$ (Figure 8).

It can be concluded from Figure 8 that with the decrease of the bulk modulus, the pore tends to be easily deformed, resulting in a significant decrease of the pore diameter, contact angle, and a slight diminution of the interfacial tension. The capillary force can be increased with the 


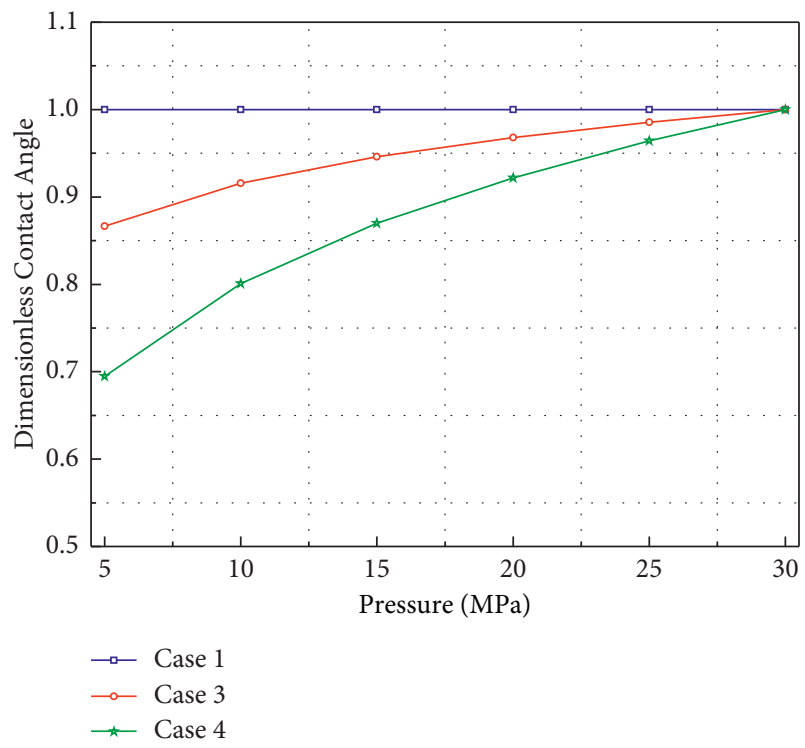

FIGURE 3: Variation of dimensionless contact angle with pressure under different cases.

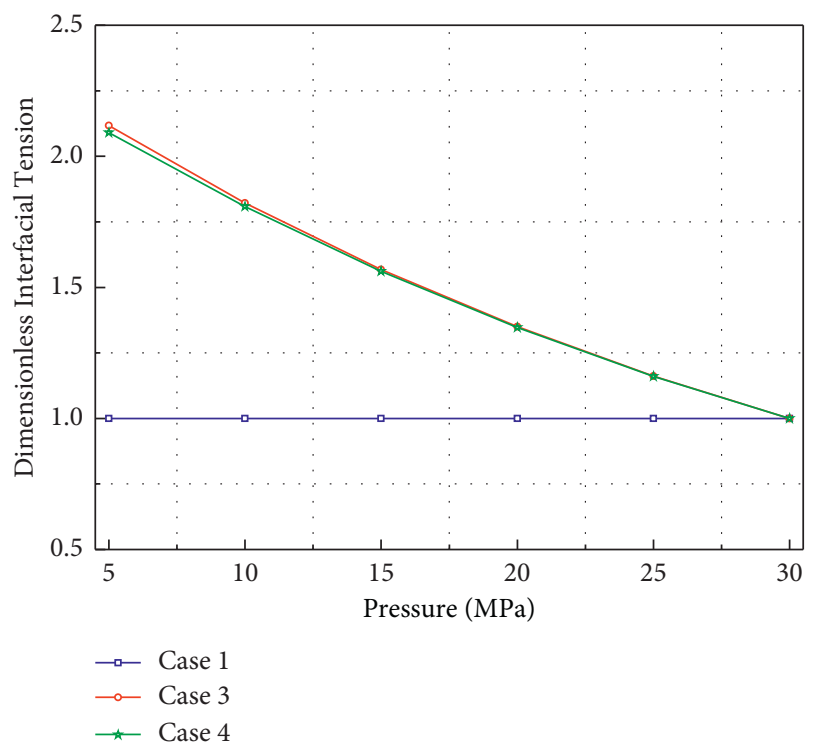

Figure 4: Variation of dimensionless interfacial tension with pressure under different cases.

increase of the above factors. And thus, the more deformable the pores are, the more significant the dynamic capillary force effect is.

Based on that, the variation of fluid flow with bulk modulus under two production modes is furtherly analyzed, and the results are shown in Figure 9. Mode 1 in Figure 9 is the oil-in-water and mode 2 is the water-drive-oil, respectively. Moreover, the dimensionless flow rate is defined as the ratio between the flow rate under the pressure and the flow rate under the initial pressure.

As seen from Figure 9, the oil-water dimensionless flow rates under the oil-in-water and water-drive-oil transport

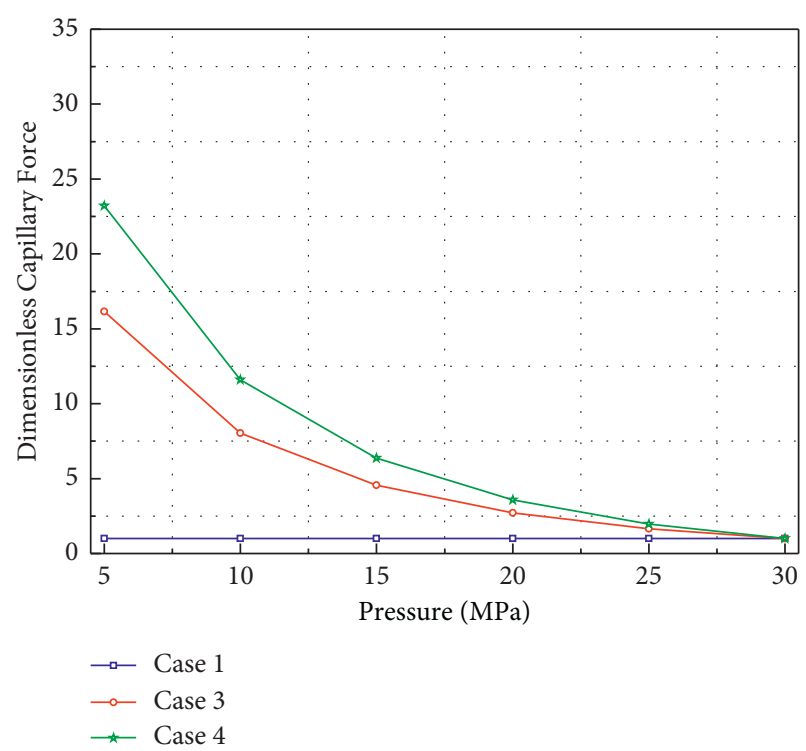

FIGURE 5: Variation of dimensionless capillary force with pressure under different conditions.

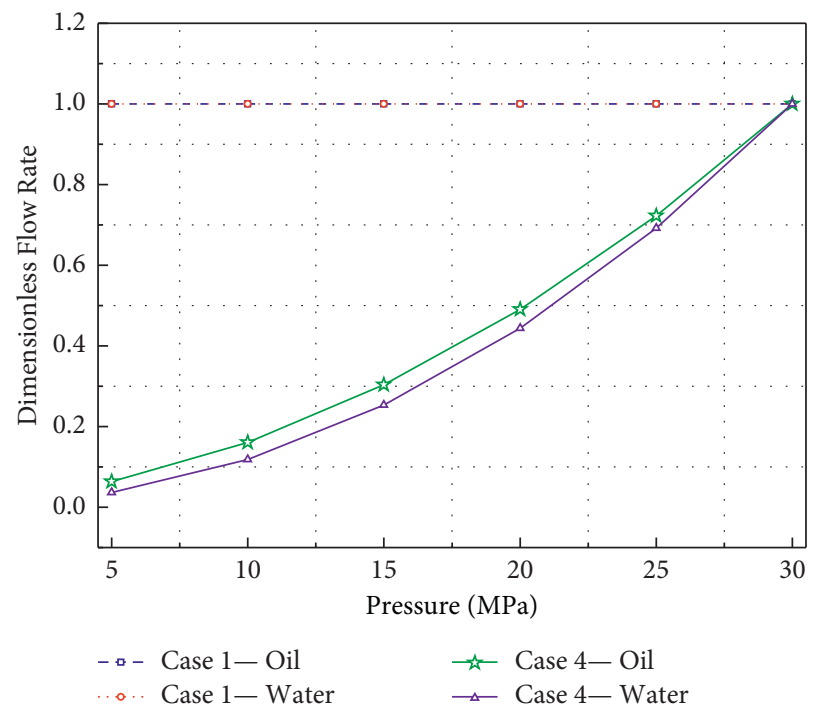

FIGURE 6: Variation of dimensionless flow rate with pressure under different conditions (oil-in-water mode).

modes decrease rapidly with the decrease of the bulk modulus, which demonstrate that the well's production can be greatly decreased due to deformable pores when the parameters in Table 1 are used.

5.3. Influence of Transport Modes. The variations of dimensionless flow rate with pressure under the oil-in-water and water-drive-oil modes are calculated through equations (39), (41), and (48), respectively, as shown in Figure 10.

As revealed from the comparison in Figure 10, for the oil-in-water transport mode, the flow rate of the water phase decreases more rapidly with the drop of the pore pressure than that of the oil phase, which will lead to a gradual 


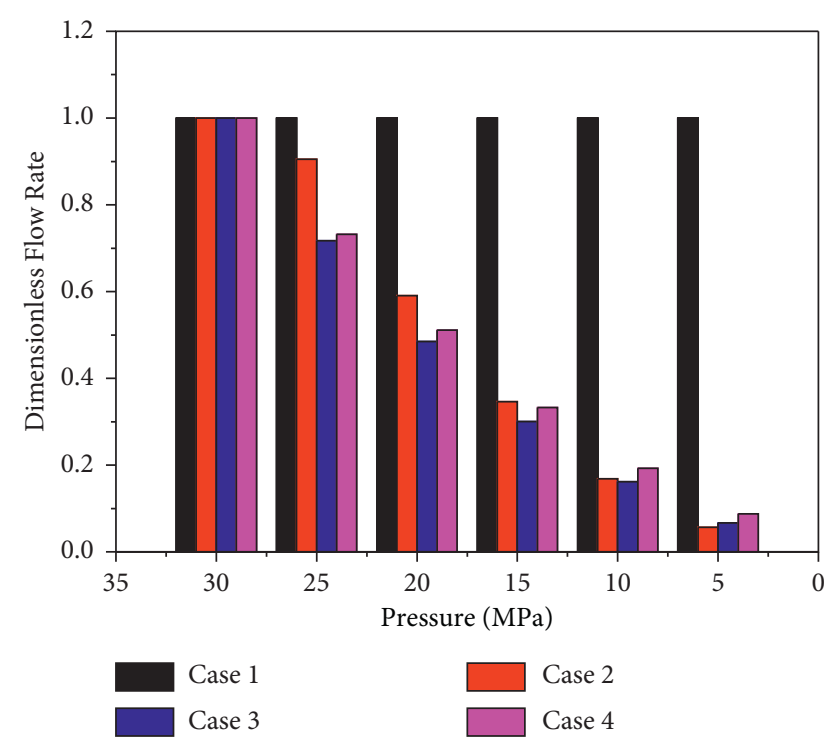

Figure 7: Relationship between dimensionless flow rate and pressure under different cases (water-drive-oil mode).

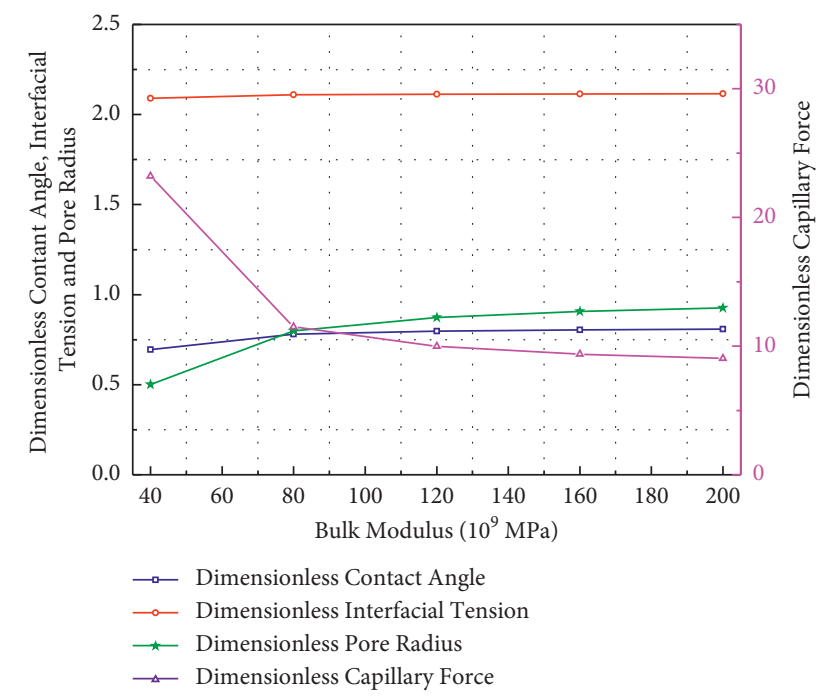

Figure 8: Variation of dimensionless contact angle, interfacial tension, pore diameter, and capillary force with bulk modulus.

decrease in water cut. Meanwhile, the reduction of flow rate for the water-drive-oil mode is smaller than that of the oilin-water mode, which is the result of the significant rise of the capillary force in nanopores for the water-drive-oil mode.

5.4. Influence of Driving Force. In the preceding analysis of this work, the driving force is assumed to be relatively large and thus the driving force plays a crucial role in the twophase flow. However, during the extraction of shale oil, the driving force may be limited. To furtherly analyze the influence of the pore pressure on the two-phase flow rate under small driving force, equation (48) can be used to calculate and determine the relationship between flow rate

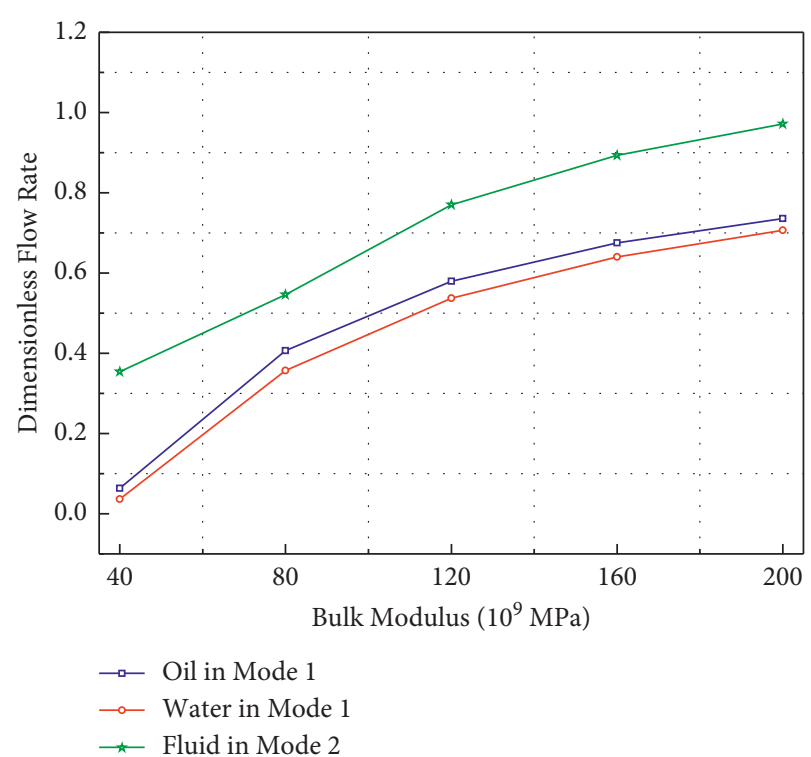

FIGURE 9: Variation of dimensionless flow rate with bulk modulus (mode 1 is the oil-in-water, mode 2 is the water-drive-oil).

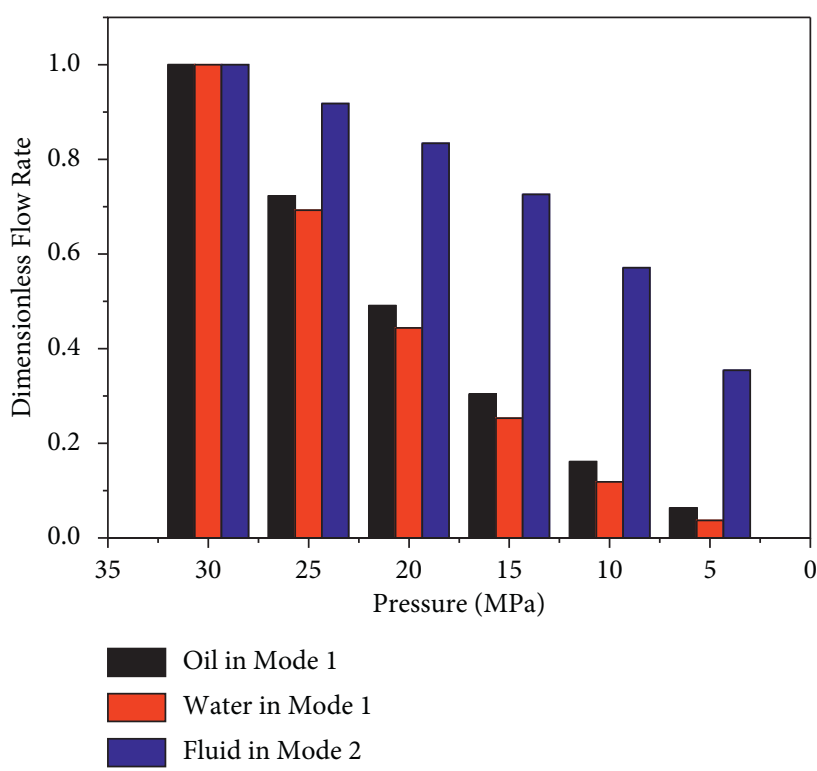

FIgURE 10: The correlation between dimensionless flow rate and pressure under different modes.

and pore pressure with different driving forces, as shown in Figure 11.

According to Figure 11, when the driving force is relatively large (0.2 $\mathrm{MPa})$, the seepage channel decreases significantly with the drop of the pore pressure, which is due to the pore deformation, and then the flow rate can be greatly decreased.

However, for a smaller driving force condition (0.1 MPa), the effect of the capillary force on the flow rate turns to be more remarkable. When the pore pressure ranges from $20 \mathrm{MPa}$ to $30 \mathrm{MPa}$, the decrease of the pore pressure will lead to the narrowing of seepage channels and then the 


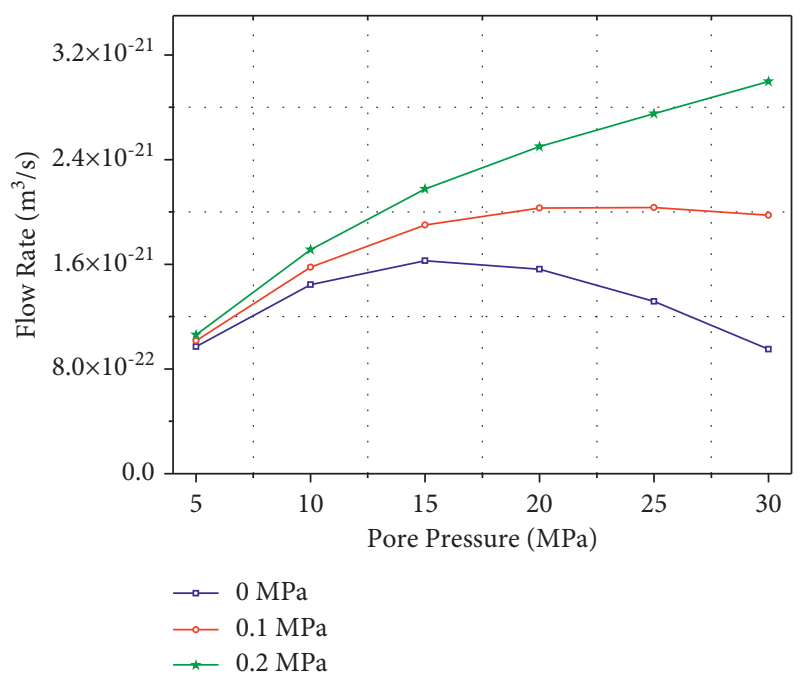

FIGURE 11: The correlation between flow rate and pore pressure for different driving forces.

capillary force can be increased, which can lead to the small velocity change in this range. When the pore pressure is furtherly decreased, the effect of seepage channel variation on the flow rate is more significant although the capillary force increases rapidly, which leads to the decreasing of the flow velocity when the pore pressure ranges from $5 \mathrm{MPa}$ to $20 \mathrm{MPa}$.

When we assume that the driving force is neglected $(0 \mathrm{MPa})$, the flow velocity increases firstly and then decreases with the decreasing of the pore pressure. The maximum flow velocity is obtained when the pore pressure equals to $15 \mathrm{MPa}$. This phenomenon is due to the competitive effect of the decreasing of the seepage channel and the increasing of the capillary force caused by the reduction of the pore pressure. Suppose that the driving force during the extraction of shale oil is usually small or even close to zero, an optimal pressure to optimize the influence of seepage channel and capillary force usually exists. Accordingly, special attention should be paid to pressure control to improve the development efficiency of shale oil.

\section{Conclusions}

Based on the above analysis and discussions, the main conclusions in this work can be obtained as follows:

(1) By using the Gassmann method, combined with microscopic deformation theory, a mathematical model for the variation of nanopore diameter and surface roughness with pore pressure is established, which lays a solid foundation on the dynamic pore characteristics and its effect on the inter-phase interaction as well as transport mechanisms.

(2) Taking the co-effect of the pore size, pressure, and surface roughness on the wettability and the interfacial tension into consideration, the mathematical models of dynamic capillary forces under the direct or indirect effect on pressure variations can be furtherly constructed.

(3) Based on the microscopic pore deformation and the dynamic capillary force characterization, mathematical models of two-phase flow under the oil-inwater and water-drive-oil mode in shale nanopores can be derived to lay a basis on the control factor analysis.

(4) The single effect of the pore deformation leads to a decrease in the interfacial tension while the capillary force significantly increases due to the pore deformation. Therefore, the dynamic capillary force phenomenon under the co-effect of the pore deformation and pressure variations is more comprehensive.

(5) The pore deformation significantly hinders the fluid transport for the oil-in-water mode. However, the influence of the capillary force caused by the pore deformation turns to be more significant with the reduction of the driving force. During the withdrawal of shale oil, the driving force is usually limited. Therefore, an optimal pressure can be found to balance the effect of the seepage channel and capillary force.

(6) The principal aim of this paper was to investigate the two-phase flow in the larger nanopores without considering the significant velocity slip. It is suggested to further combine the multi-component microscopic interaction at the molecular scale and velocity slip in future and thus the migration mechanisms in the smaller nano-pores can be discussed in detail.

\section{Nomenclature}

Latin Symbols

E: $\quad$ Compression modulus, $\mathrm{MPa}$

$E_{s}: \quad$ Compression modulus, $\mathrm{MPa}$

$E_{0}$ : Enthalpy of vaporization, $\mathrm{kJ} / \mathrm{mol}$

G: $\quad$ Pore shape correction coefficient, dimensionless

$K_{s}$ : Bulk modulus, $\mathrm{MPa}$

L: $\quad$ Pore length, $\mathrm{m}$

$L_{2}$ : $\quad$ Length of free water zone, $\mathrm{m}$

$p_{1}$ : $\quad$ Starting pressure, $\mathrm{MPa}$

$p_{2}$ : $\quad$ Ending pressure, $\mathrm{MPa}$

$p_{1}^{\prime}$ : $\quad$ Pressure on the left side of the bend level, $\mathrm{MPa}$

$p_{2}^{\prime}$ : $\quad$ Pressure on the left side of the bend level, $\mathrm{MPa}$

$q_{o}$ : Oil flow rate, $\mathrm{m}^{3} / \mathrm{s}$

$q_{w}: \quad$ Water flow rate, $\mathrm{m}^{3} / \mathrm{s}$

$R: \quad$ Ideal gas constant, dimensionless

$r_{i}$ : The pore radius under the initial formation pressure condition, $\mathrm{nm}$

$r_{o-\text { eff }}:$ Effective radius for oil flow, $\mathrm{m}$

$r_{w-\mathrm{eff}}$ : Effective radius for water flow, $\mathrm{m}$

$S_{o}: \quad$ Oil saturation, dimensionless

$T_{b}$ : Boiling point, $\mathrm{K}$

$V_{\phi-r}$ : Pore volume for the certain pressure, $\mathrm{nm}^{3}$ 
Greek Symbols

$\alpha$ : The Bolt Coefficient, dimensionless

$\varepsilon_{s}$ : Solid skeleton deformation ratio, dimensionless

$\varepsilon_{t}$ : Porous media volume deformation ratio, dimensionless

$\varepsilon_{\phi}$ : Pore volume deformation ratio, dimensionless

$\theta$ : Contact angle for certain pore radius

$\theta_{e}$ : Intrinsic contact angle of the solid surface

$\theta_{i}$ : Contact angle for the initial condition

$\mu_{o}:$ Viscosity for oil, $\mathrm{cp}$

$\mu_{w}$ : Viscosity for water, cp

$\xi$ : Interfacial modulus, dimensionless

$v$ : Poisson's ratio, dimensionless

$\phi$ : Porosity, dimensionless

$\lambda$ : Period distance of protrusion, $\mathrm{nm}$

\section{Others}

$\Delta a:$ Change of rough protrusion height, $\mathrm{nm}$

$\Delta p_{f}$ : Change of fluid pressure, $\mathrm{MPa}$

$\Delta p_{o}$ : Pressure drop of oil, $\mathrm{MPa}$

$\Delta p_{w}$ : Pressure drop of water, $\mathrm{MPa}$

$\Delta V_{\phi}$ : Change of pore volume, $\mathrm{nm}^{3}$

$\Delta \bar{\sigma}: \quad$ Average normal stress acting on the pore-bearing rock, $\mathrm{MPa}$.

\section{Data Availability}

All data included in this study are available upon request from the corresponding author.

\section{Conflicts of Interest}

The authors declare no conflicts of interest.

\section{Acknowledgments}

The authors would like to appreciate the financial support of the National Natural Science Foundation of China (No. 52004038), Study on the Microscopic Phase Behavior and Migration Mechanism of $\mathrm{CO}_{2}$ and Multicomponent Alkanes in Shale Dynamical Nanopore. The authors also would like to appreciate the support of the Natural Science Research General project of Jiangsu Province (No. 20KJB440003), Study on carbon dioxide-methane transport mechanism of coal seam nanopores with multiple inducement deformation fields.

\section{References}

[1] S. Wang, F. Javadpour, and Q. Feng, "Molecular dynamics simulations of oil transport through inorganic nanopores in shale," Fuel, vol. 171, pp. 74-86, 2016.

[2] J. Cui, Q. Sang, Y. Li, C. Yin, Y. Li, and M. Dong, "Liquid permeability of organic nanopores in shale: calculation and analysis," Fuel, vol. 202, pp. 426-434, 2017.

[3] Y. L. Su, M. J. Lu, M. Li, W. Wang, and M. Dong, "Numerical simulation of coupled flow of multiple porous media in shale reservoir," Oil and Gas Geology, vol. 40, no. 3, pp. 645-652+660, 2019.

[4] Y. Liu, J. Zeng, G. Yang et al., "An innovative method for the characterization of oil content in lacustrine shale-oil systems: a case study from the middle permian lucaogou formation in the Jimusaer Sag, Junggar Basin," Marine and Petroleum Geology, vol. 130, 2021.

[5] S. N. Saltykova, "Microanalysis of oil shale of the leningrad field,” Key Engineering Materials, vol. 854, pp. 188-193, 2020.

[6] B. R. Crawford, J. A. Freysteinson, and G. Gupta, "Measuring pressure dependent permeability in shales: impact of stress path and anisotropy," in Proceedings of the 53rd US Rock Mechanics/Geomechanics Symposium, American Rock Mechanics Association, New York City, NY, USA, June 2019.

[7] S. Das, J. Adeoye, I. Dhiman, H. Z. Bilheux, and B. R. Ellis, "Imbibition of mixed charge surfactant fluids in shale fractures," Energy \& Fuels, vol. 33, pp. 2839-2847, 2019.

[8] S. Gruener and P. Huber, "Capillarity-driven oil flow in nanopores: darcy scale analysis of lucas-washburn imbibition dynamics," Transport in Porous Media, vol. 126, no. 3, pp. 599-614, 2019.

[9] W. F. Zhou, X. Wang, X. G. Lu, H. Pan, T. T. Wang, and D. W. Han, "Dynamic imbibition oil recovery effect and its influencing factors in tight oil reservoir," Daqing Petroleum Geology and Development, vol. 36, no. 003, pp. 148-155, 2017.

[10] N. Wijaya and J. J. Sheng, "Shut-in effect in removing water blockage in shale-oil reservoirs with stress-dependent permeability considered," SPE Reservoir Evaluation \& Engineering, vol. 23, no. 1, pp. 81-94, 2019.

[11] B. Y. Kim, I. Y. Akkutlu, V. Martysevich, and R. Dusterhoft, "Monolayer microproppant-placement quality using splitcore-plug permeability measurements under stress," SPE Journal, vol. 24, no. 4, pp. 1790-1808, 2019.

[12] T. Stoxreiter, P. Gehwolf, and R. Galler, "Alternative approaches for the determination of unconfined rock deformation and strength properties," Rock Mechanics and Rock Engineering, vol. 53, no. 1, pp. 411-433, 2020.

[13] J. B. Wang, Three-dimensional Reconstruction of Rock Pore Structure and Numerical Simulation of Micro- and Microtransport, China University of Mining, Beijing, China, 2014.

[14] G. Lei, P. C. Dong, S. Yang, B. Wang, and S. Y. Mo, “Analysis of stress sensitivity of low permeability reservoir based on the arrangement of rock particles," Rock and Soil Mechanics, vol. 35, no. s1, pp. 209-214, 2014.

[15] Y. Xia, Y. Jing, and M. Chen, "Multi-field coupling mechanism in the process of shale gas transport," Science in China: Physics, Mechanics, Astronomy, vol. 45, no. 9, p. 94703, 2015.

[16] D. B. Bennion and S. Bachu, "The impact of interfacial tension and pore size distribution/Capillary force character on $\mathrm{CO}_{2}$ relative permeability at reservoir conditions in $\mathrm{CO}_{2}$-brine systems," in Proceedings of the SPE/DOE Symposium on Improved Oil recovery, pp. S416-S417, Tulsa, OK, USA, April 2006.

[17] A. Najafi-Marghmaleki, A. Barati-Harooni, A. Soleymanzadeh, S. J. Samadi, B. Roshani, and A. Yari, "Experimental investigation of effect of temperature and pressure on contact angle of four Iranian carbonate oil reservoirs," Journal of Petroleum Science and Engineering, vol. 142, pp. 77-84, 2016.

[18] S. Tian, S. Lu, H. Xue, L. Xie, and J. Li, "The influence of pore throat radius on its internal oil and water wettability," Acta Geologica Sinica-English Edition, vol. 89, no. s1, pp. 166-167, 2015.

[19] A. Barati-Harooni, A. Soleymanzadeh, A. Tatar et al., "Experimental and modeling studies on the effects of temperature, pressure and brine salinity on interfacial tension in live oil-brine systems," Journal of Molecular Liquids, vol. 219, pp. 985-993, 2016. 
[20] G. R. Jerauld and S. J. Salter, "The effect of pore-structure on hysteresis in relative permeability and Capillary force: porelevel modeling," Transport in Porous Media, vol. 5, no. 2, pp. 103-151, 1990.

[21] J. Ansarinasab and M. Jamialahmadi, "Investigating the effect of interfacial tension and contact angle on capillary pressure curve, using free energy Lattice Boltzmann Method," Journal of Natural Gas Science and Engineering, vol. 35, pp. 1146-1157, 2016.

[22] X. Han, X. Jiang, L. Yu, and Z. Cui, "Change of pore structure of oil shale particles during combustion. Part 1. Evolution mechanism," Energy \& Fuels, vol. 20, no. 6, pp. 2408-2412, 2006.

[23] M. Liang, Z. Wang, C. Li, and H. Li, "Evolution of pore structure in gas shale related to structural deformation," Fuel, vol. 197, pp. 310-319, 2017.

[24] F. Shang, Y. Zhu, H. Gao, Y. Wang, and R. Liu, "Relationship between tectonism and composition and pore characteristics of shale reservoirs," Geofluids, vol. 2020, Article ID 9426586, 14 pages, 2020.

[25] M. Arif, Y. Zhang, and S. Iglauer, "Shale wettability: data sets, challenges, and outlook," Energy \& Fuels, vol. 35, pp. 2965-2980, 2021.

[26] H. Sharifigaliuk, S. M. Mahmood, W. Al-Bazzaz, and V. Khosravi, "Complexities driving wettability evaluation of shales toward unconventional approaches: a comprehensive review," Energy \& Fuels, vol. 35, no. 2, 2021.

[27] B. Khodadadi, M. Siddiqui, M. A. Pirzada, F. Le-Hussain, S. Iglauer, and H. Roshan, "Direct observation of two-phase flow in deformable fractures of shales: a utica shale example," Journal of Petroleum Science and Engineering, vol. 194, pp. 107-487, 2020.

[28] F. Zhang and H. Emami-Meybodi, "A semi-analytical method for two-phase flowback rate-transient analysis in shale gas reservoirs," SPE Journal, vol. 25, pp. 1599-1622, 2020.

[29] T. Zhang, F. Javadpour, J. Li, Y. L. Zhao, L. H. Zhang, and X. F. Li, "Pore-scale perspective of gas/water two-phase flow in shale," SPE Journal, vol. 26, no. 2, pp. 1-19, 2021.

[30] S. Rafieepour, S. Zamiran, and M. Ostadhassan, "A cost-effective chemo-thermo-poroelastic wellbore stability model for mud weight design during drilling through shale formations," Journal of Rock Mechanics and Geotechnical Engineering, vol. 12, no. 4, 2020.

[31] C. R. Clarkson, B. Yuan, Z. Zhang et al., "Evaluation of the impact of multi-phase flow on reservoir signatures in the wolfcamp shale," Journal of Natural Gas Science and Engineering, vol. 76, no. 5, pp. 103-187, 2020.

[32] M. M. Carroll and N. Katsube, "The role of terzaghi effective stress in linearly elastic deformation," Journal of Energy Resources Technology, vol. 105, no. 4, pp. 509-511, 1983.

[33] H. M. Lu and Q. Jiang, "Size-dependent surface tension and Tloman's length of droplets," Langmuir, vol. 21, no. 2, pp. 779-781, 2005. 